\title{
A generalizable definition of chemical similarity for read-across
}

\author{
Matteo Floris ${ }^{1,2 \dagger}$, Alberto Manganaro ${ }^{3 \dagger}$, Orazio Nicolotti ${ }^{4}$, Ricardo Medda ${ }^{2}$, Giuseppe Felice Mangiatordi ${ }^{4}$ \\ and Emilio Benfenati ${ }^{3^{*}}$
}

\begin{abstract}
Background: Methods that provide a measure of chemical similarity are strongly relevant in several fields of chemoinformatics as they allow to predict the molecular behavior and fate of structurally close compounds. One common application of chemical similarity measurements, based on the principle that similar molecules have similar properties, is the read-across approach, where an estimation of a specific endpoint for a chemical is provided using experimental data available from highly similar compounds.

Results: This paper reports the comparison of multiple combinations of binary fingerprints and similarity metrics for computing the chemical similarity in the context of two different applications of the read-across technique.

Conclusions: Our analysis demonstrates that the classical similarity measurements can be improved with a generalizable model of similarity. The proposed approach has already been used to build similarity indices in two open-source software tools (CAESAR and VEGA) that make several QSAR models available. In these tools, the similarity index plays a key role for the assessment of the applicability domain.
\end{abstract}

Keywords: Chemical similarity, Read-across, Applicability domain, QSAR

\section{Background}

Methods that provide a measure of similarity between chemical compounds are becoming increasingly important, as several fields of chemoinformatics are in need of automated tools for the quick retrieval of congeneric molecules, thereby avoiding the use of human experts for the highly time demanding burden of checking every single compound and of evaluating its similarity with respect to a given reference [1]. Such a task is more challenging or even unfeasible when dealing with large-sized database comprising thousands of compounds.

To date, several approaches and algorithms for calculating chemical similarity have been developed [2,3]. However, a still open and debated issue behind such different approaches is precisely in the concept of similarity. It is not possible to define in an unambiguous way (and, consequently, with an unambiguous algorithm) how similar two chemical entities are. In fact, two compounds can be seen as more or less similar with respect to the

\footnotetext{
*Correspondence: emilio.benfenati@marionegri.it

${ }^{\dagger}$ Equal contributors

${ }^{3}$ IRCCS-Istituto di Ricerche Farmacologiche Mario Negri, Milan, Italy

Full list of author information is available at the end of the article
}

chemical features taken into consideration or chosen as a priority. For instance, if a similarity measurement is needed for QSAR purposes, the same molecular descriptors (i.e. physicochemical substituent representing hydrophobic, electronic and steric effects) used for deriving the QSAR model could be used. However, in other circumstances, the similarity could rely on holistic approaches based on a broader description of the chemical structure.

Another point leading to different approaches is related to practical applications of the similarity measurement. Excessive complexity must be avoided to obtain algorithms that can be calculated in a reasonable time.

The binary fingerprint approach is probably one of the most used methods to evaluate similarity $[4,5]$. It is a milestone example of an acceptable trade-off between the wealth of information encoded and the chance of performing an easy and quick comparison of a large molecular data set.

A fingerprint consists of a fixed length string of bits in which the occurrence of molecular fragments is encoded (as one or more bits set to 1 ) by a hashing algorithm. The encoded sets of bits for different fragments could share one or more bits, so each bit of the fingerprint does not 
represent a unique structural feature (also meaning that it is not possible to generate the set of original fragments from a fingerprint). Fingerprints of two molecules can be compared to quantify (dis)similarity using some distance measure. A popular example is the Tanimoto index [6]. Structural keys represent a related approach: the string is not built with a hashing algorithm, but each bit represents an a-priori defined structural feature [7].

Fingerprints and structural keys are really useful for fast matching of similar structures and have been largely used for screening large molecular databases. Nevertheless, they suffer of some drawbacks $[8,9]$. For instance, they encode the presence or absence of certain fragments or functional groups without accounting of their actual occurrence per compound (i.e. the number of times each fragment or function groups is found in the same molecule). This can lead to inaccurate matching, and thus can return artifacts.

Several binary fingerprints are available. Among others, fragment-based Daylight [10] and Tripos UNITY 2D fingerprints [11] are some of the best known commercial examples.

In the present work, we decided to focus our attention only on the fingerprints available in the Chemistry Development Kit $[12,13]$, which are free and open source implementations of different fingerprint algorithms.

Furthermore, several similarity coefficients are available; a comprehensive and up-to-date list has been recently summarized by Todeschini et al. [14] and used in the present work to choose similarity coefficients to be tested. Remarkably, Todeschini listed 51 similarity coefficients for binary variables extracted from the literature and compared using both simulated and real data.

Our aim is that of exploring the possibility of blending fingerprints with non-binary structural keys based on constitutional molecular descriptors. The basic idea is that such a combination can help to overcome the drawbacks of a plain fingerprint approach and thus to increase the accuracy of similarity measurements, yet avoiding an excessive calculation complexity. In this respect, we developed an integrated similarity index resulting from the weighted combination of a fingerprint array and three structural keys based on molecular descriptors. We then designed a batch process to evaluate the performances of different fingerprints, different similarity coefficients, and different weighting schemes for the elements contained in the final index.

We chose to use, for the batch process, a read-across approach on two distinct datasets, in order to find an acceptable criterion of choice of elements and weighting scheme for the similarity index in a generic application. Our efforts resulted in a comparative analysis of the performances on the two datasets of all the possible combinations of 9 fingerprint implementations and 44 similarity coefficients, followed by an exploration of a reasonable subset of all the possible weighting schemes for the fingerprint and the structural keys based on molecular descriptors.

A scheme providing good performances on both datasets has finally been chosen to build the similarity index, actually implemented in the VEGA platform [15] (an opensource on-line platform providing several QSAR models).

\section{Methods}

\section{Fingerprints}

We decided to evaluate the performance of 9 different fingerprint algorithms, which are implemented in the Chemistry Development Kit (CDK) libraries. While they fall under the generic definition of fingerprints, some of them are structural keys and not hashing-based fingerprints. More specifically, the fingerprints here considered are the following:

1. Default Fingerprints (as defined by Daylight [10]),

2. Extended Fingerprints (same as Default, but with additional bits that take into account ring features),

3. Graph-only fingerprints (same as Default, but do not take bond orders into account),

4. Hybridization fingerprints (same as Default, but do not perform aromaticity perception),

5. E-State fragments (79 bit fingerprints described by Kier and Hall [16]),

6. Klekota-Roth fingerprints (set of 4860 chemical substructures enriched for biological activity [17]),

7. MACCS keys (structural key made of a set of 166 bits [18]),

8. Pubchem fingerprints (structural key made of 881 keys [19]),

9. Substructure fingerprints (structural key made of 307 bits [20].

\section{Molecular descriptors based structural keys}

We decided to build three structural keys made of molecular descriptors related to constitutional issues. The hypothesis that lead to these keys was to test if such information could be successfully coupled with the use of fingerprints, so that these keys can fill the information gap of fingerprints. As these keys are made of molecular descriptors, they are no longer binary keys. The descriptors used for these keys were calculated by an in-house JAVA software module, based on CDK libraries; for the definition of the descriptors the commercial software Dragon [21] has been taken as reference.

The three keys are:

- Constitutional descriptors (CD): this key is made of 35 constitutional descriptors, as reported in Table 1

- Hetero-atoms descriptors (HD): this key is made of 11 counters for different types of hetero-atoms, as 


\begin{tabular}{|c|c|}
\hline Name & Description \\
\hline MW & Molecular weight \\
\hline AMW & Average molecular weight \\
\hline Sv & Sum of atomic van der Waals volumes \\
\hline Mv & Mean atomic van der Waals volum \\
\hline $\mathrm{Sp}$ & Sum of atomic polarizabilities \\
\hline Mp & Mean atomic polarizability \\
\hline Se & Sum of atomic Sanderson electronegativities \\
\hline Me & Mean atomic Sanderson electronegativity \\
\hline nAt & Number of atoms \\
\hline$n S k$ & Number of non-H atoms \\
\hline$n B t$ & Number of bonds \\
\hline$n B o$ & Number of non-H bonds \\
\hline$n B m$ & Number of multiple bonds \\
\hline nDblBo & Number of double bonds \\
\hline nTrpBo & Number of triple bonds \\
\hline nArBo & Number of aromatic bonds \\
\hline SCBO & Sum of conventional bond orders ( $\mathrm{H}$-depleted) \\
\hline $\mathrm{nH}$ & Number of Hydrogen atoms \\
\hline $\mathrm{nC}$ & Number of Carbon atoms \\
\hline $\mathrm{nN}$ & Number of Nitrogen atoms \\
\hline $\mathrm{nO}$ & Number of Oxygen atoms \\
\hline$n P$ & Number of Phosphorous atoms \\
\hline nS & Number of Sulfur atoms \\
\hline$n F$ & Number of Fluorine atoms \\
\hline $\mathrm{nCl}$ & Number of Chlorine atoms \\
\hline$n B r$ & Number of Bromine atoms \\
\hline $\mathrm{nl}$ & Number of lodine atoms \\
\hline$n B$ & Number of Boron atoms \\
\hline HPerc & Percentage of $\mathrm{H}$ atoms \\
\hline CPerc & Percentage of $\mathrm{C}$ atoms \\
\hline NPerc & Percentage of $\mathrm{N}$ atoms \\
\hline OPerc & Percentage of $\mathrm{O}$ atoms \\
\hline XPerc & Percentage of halogen atoms \\
\hline nHet & Number of heteroatoms \\
\hline$n X$ & Number of halogen atoms \\
\hline
\end{tabular}

reported in Table 2. These descriptors are a subset of the constitutional descriptors. We chose to build a key with this subset in order to have the possibility of giving it different weights so to remark the feature it represent in the computation of chemical similarity. This stems from the observation that often the generic idea of chemical similarity is strongly influenced by small differences in the number and type of heteroatoms, i.e. molecules with several similar
Table 2 Descriptors in the hetero-atoms descriptors (HD) key

\begin{tabular}{ll}
\hline Name & Description \\
\hline $\mathrm{nN}$ & Number of Nitrogen atoms \\
$\mathrm{nO}$ & Number of Oxygen atoms \\
$\mathrm{nP}$ & Number of Phosphorous atoms \\
$\mathrm{nS}$ & Number of Sulfur atoms \\
$\mathrm{nF}$ & Number of Fluorine atoms \\
$\mathrm{nCl}$ & Number of Chlorine atoms \\
$\mathrm{nBr}$ & Number of Bromine atoms \\
$\mathrm{nl}$ & Number of lodine atoms \\
$\mathrm{nB}$ & Number of Boron atoms \\
$\mathrm{nHet}$ & Number of heteroatoms \\
$\mathrm{nX}$ & Number of halogen atoms \\
\hline
\end{tabular}

features (molecular weight, number and type of rings, bonds etc.) can be considered remarkably different just because they differ in the presence/absence of some heteroatoms.

- Functional Groups (FG): this key is made of 154 functional groups, as defined in Dragon.

\section{Similarity coefficients}

We built two sets of similarity coefficients to be tested respectively with the chosen fingerprints (binary coefficients) and descriptors based keys (non-binary coefficients). The chosen binary coefficients are 44, reported in Table 3, coming from the work of Todeschini et al. [14]. The chosen non-binary coefficients are 6 , reported in Table 4, coming from the work of Holliday [22]. All the coefficients have been implemented in an in-house JAVA software module.

\section{Similarity index}

In order to combine the fingerprint with the descriptors based keys, we designed a generic scheme for the similarity index SI, defined as follow:

$$
\begin{aligned}
\mathrm{SI}(\mathrm{A}, \mathrm{B})=[ & \left.\mathrm{Sb}\left(\mathrm{FP}_{\mathrm{a}}, \mathrm{FP}_{\mathrm{b}}\right)\right] \mathrm{W}_{\mathrm{fp}} *\left[\mathrm{Snb}\left(\mathrm{CD}_{\mathrm{a}}, \mathrm{CD}_{\mathrm{b}}\right)\right] \mathrm{W}_{\mathrm{cd}} \\
& *\left[\mathrm{Snb}\left(\mathrm{HD}_{\mathrm{a}}, \mathrm{HD}_{\mathrm{b}}\right)\right] \mathrm{W}_{\mathrm{hd}} \\
& *\left[\mathrm{Snb}\left(\mathrm{FG}_{\mathrm{a}}, \mathrm{FG}_{\mathrm{b}}\right)\right] \mathrm{W}_{\mathrm{fg}}
\end{aligned}
$$

where:

$\mathrm{A}$ and $\mathrm{B}$ are two molecules to be compared;

$\mathrm{FP}_{\mathrm{a}}, \mathrm{CD}_{\mathrm{a}}, \mathrm{HD}_{\mathrm{a}}, \mathrm{FG}_{\mathrm{a}}, \mathrm{FP}_{\mathrm{b}}, \mathrm{CD}_{\mathrm{b}}, \mathrm{HD}_{\mathrm{b}}, \mathrm{FG}_{\mathrm{b}}$ are the Fingerprint, Constitutional Descriptors, Heteroatom Descriptors and Functional Groups keys as defined before, respectively calculated on the two molecules A and B; 
Table 3 Binary similarity coefficients

\begin{tabular}{|c|c|c|c|}
\hline No. & Name & No. & Name \\
\hline 1 & Simple matching & 23 & Dennis \\
\hline 2 & Rogers/Tanimoto & 24 & Cole 1 \\
\hline 3 & Jaccard/Tanimoto & 25 & Cole 2 \\
\hline 4 & Gleason/Dice/Sorensen/Nei-Li & 26 & Dispersion \\
\hline 5 & Russel-Rao & 27 & Goodman-Kruskal \\
\hline 6 & Forbes & 28 & Sokal-Sneath 3 \\
\hline 7 & Simpson & 29 & Sokal-Sneath 4 \\
\hline 8 & Braun-Blanquet & 30 & Phi \\
\hline 9 & Driver-Kroeber/Ochiai & 31 & Dice 1 \\
\hline 10 & Baroni-Urbani 1 & 32 & Dice 2 \\
\hline 11 & Kulczynski 1 & 33 & Sorgenfrei \\
\hline 12 & Sokal-Sneath 1 & 34 & Cohen \\
\hline 13 & Sokal-Sneath 2 & 35 & Peirce 1 \\
\hline 14 & Jaccard 2 & 36 & Peirce 2 \\
\hline 15 & Faith & 37 & Maxwell-Pilliner \\
\hline 16 & Mountford & 38 & Harris-Lahey \\
\hline 17 & Michael & 39 & CT1 \\
\hline 18 & Rogot-Goldberg & 40 & $\mathrm{CT} 2$ \\
\hline 19 & Hawkins-Dotson & 41 & CT3 \\
\hline 20 & Yule 1 & 42 & $\mathrm{CT} 4$ \\
\hline 21 & Yule 2 & 43 & CT5 \\
\hline 22 & Fossum & 44 & Austin-Colwell angular coeff. \\
\hline
\end{tabular}

The number of each coefficient is the same as in the paper by Todeschini et al.

$\mathrm{Sb}\left(\mathrm{X}_{\mathrm{a}}, \mathrm{X}_{\mathrm{b}}\right)$ is the result of the application of a binary similarity coefficient to two fingerprints $X_{a}$ and $X_{b}$, where the resulting values are in the interval $[0,1]$;

$\operatorname{Snb}\left(X_{a}, X_{b}\right)$ is the result of the application of a nonbinary similarity coefficient to two descriptors based keys $X_{a}$ and $X_{b}$, where the resulting values are in the interval $[0,1]$

$\mathrm{W}_{\mathrm{fp}}, \mathrm{W}_{\mathrm{cd}}, \mathrm{W}_{\mathrm{hd}}, \mathrm{W}_{\mathrm{fg}}$ are the relative weights of the four contributions, under the condition:

$$
\mathrm{W}_{\mathrm{fp}}+\mathrm{W}_{\mathrm{cd}}+\mathrm{W}_{\mathrm{hd}}+\mathrm{W}_{\mathrm{fg}}=1
$$

As it can been seen, the proposed index simply takes into account the different contribution of the similarity

Table 4 Non-binary similarity coefficients

\begin{tabular}{lll}
\hline No. & Name & Code \\
\hline 1 & Mean Camberra & MC \\
2 & Divergence & Div \\
3 & Bray/Curtis & BC \\
4 & Dice & Dice \\
5 & Sokal/Sneath & SS1 \\
6 & Cosine/Ochiai & Cos \\
\hline
\end{tabular}

The code of each coefficient is the same as in the paper by Holliday et al. (calculated with the chosen coefficient), each one with a given weight.

\section{Datasets and read-across model}

We chose two publicly available datasets from the VEGA project. The bioconcentration factor in fish (BCF) dataset comprises 473 compounds with the experimental $\mathrm{BCF}$ values. The water/octanol partition coefficient $(\log \mathrm{P})$ dataset consists of 10,005 compounds with the experimental $\log \mathrm{P}$ values.

The choice of testing the Similarity Index on these two datasets arises from the goal of finding a setting for the SI that potentially could give good performances on different kinds of data, thus implementing a "generic" idea of chemical similarity. In more detail, we focused our analysis on an endpoint with relevance for toxicity $(\mathrm{BCF})$ and on a physical-chemical property $(\log \mathrm{P})$ with several applications, furthermore having markedly different size (BCF: 860 molecules; $\log$ P 10005 molecules).

For the purpose of testing the performances of the proposed Similarity Index with different settings, we implemented in an in-house JAVA module a simple readacross based prediction model, where a property is predicted for a given compound by finding the three most similar compounds of the dataset according to the SI, and calculating the mean of their three experimental values, weighted by their SI values.

In our procedure, we calculated predictions on the basis of the leave-one-out strategy adopted for crossvalidation. Iteratively, one molecule at a time was left out of the dataset to be predicted using our read-across approach on the remaining molecules.

Finally, as the above described model approach is analogous to a regression model, we calculated the values of the coefficient of determination $\left(R^{2}\right)$ and of the root mean square error (RMSE) on all the predictions of the dataset, and used these values to quantify the quality of the model, that is directly related to how good the SI settings are.

\section{Evaluation process}

We applied a combinatorial strategy to test all the possible permutation of different settings (similarity coefficient, binary fingerprints, non-binary descriptors, weighting scheme), calculating for each of these settings the readacross model for the two datasets and the resulting $R^{2}$ and RMSE.

In a preliminary step, we processed both datasets with all the combinations of the different fingerprints and of binary similarity coefficients, for a total of about 400 permutations. At this level, we selected the best combinations (based on $\mathrm{R}^{2}$ and RMSE).

We then performed a second analysis where we used the selected couple of fingerprint/coefficient and a set of 
Table 5 Best ten results for fingerprints/similarity metrics combinations

\begin{tabular}{llllllll}
\hline FP & Metrics & BCF R $^{2}$ & BCF RMSE & LogP R & LogP RMSE & DES & UTI \\
\hline Extended & 37 & 0.546 & 0.917 & 0.775 & 0.872 & 0.970 & 0.971 \\
Extended & 34 & 0.546 & 0.919 & 0.776 & 0.870 & 0.970 \\
Extended & 18 & 0.542 & 0.922 & 0.777 & 0.869 & 0.965 \\
Pubchem & 28 & 0.541 & 0.906 & 0.772 & 0.870 & 0.963 \\
Extended & 42 & 0.534 & 0.919 & 0.780 & 0.858 & 0.963 \\
Default & 18 & 0.549 & 0.913 & 0.766 & 0.890 & 0.961 & 0.954 \\
Extended & 13 & 0.541 & 0.913 & 0.770 & 0.875 & 0.962 \\
Default & 34 & 0.549 & 0.913 & 0.765 & 0.891 & 0.954 \\
Extended & 1 & 0.540 & 0.917 & 0.770 & 0.876 & 0.953 \\
Default & 37 & 0.549 & 0.913 & 0.764 & 0.893 & 0.954 \\
\hline
\end{tabular}

FP stands for the fingerprint type, Metrics for the number (id) of the binary similarity coefficient (as reported in Table 3), for the R2 correlation coefficient, RMSE for the root mean square error, DES for the desirability function, UTI for the utility function.

combinations of the weights for the SI contributions and of non-binary similarity coefficients for the descriptors keys. We chose the following ranges for the weights:

$\mathrm{W}_{\mathrm{fp}}$ : between 0.3 and 1.0, with steps of 0.1

$\mathrm{W}_{\text {cd }}$ : between 0.0 and 0.4 , with steps of 0.05

$\mathrm{W}_{\mathrm{hd}}$ : between 0.0 and 1.0, with steps of 0.05

$\mathrm{W}_{\mathrm{fg}}$ : between 0.0 and 1.0, with steps of 0.05

under the usual condition of having the sum of weights equal to one. The batch process generated a total of about 7200 combinations of weights/coefficient.

\section{Results and discussion}

The first step has been to analyze the results of all possible permutations of fingerprint types and similarity coefficients, in order to find the best combination to be used in the following step.

We evaluated simultaneously the results by considering the values of both $R^{2}$ and RMSE, using two objectives known as utility function and desirability function for ranking the combinations on the basis of the performances on both the datasets. Such functions are usually applied in the field of multi-criteria decision making $[23,24]$. The two functions have been calculated as:

$$
\begin{aligned}
\mathrm{DES}= & \left(\mathrm{R}_{\mathrm{bcf}}\right) 0.25 *\left(\mathrm{RMSE}_{\mathrm{bcf}}\right) 0.25 \\
& *\left(\mathrm{R}_{\operatorname{logp}}\right) 0.25 *\left(\mathrm{RMSE}_{\operatorname{logp}}\right) 0.25 \\
\mathrm{UTI}= & 0.25 * \mathrm{R}_{\mathrm{bcf}}+0.25 * \mathrm{RMSE}_{\mathrm{bcf}}+0.25 \\
& * \mathrm{R}_{\operatorname{logp}}+0.25 * \mathrm{RMSE}_{\operatorname{logp}}
\end{aligned}
$$

where $R_{b c f}$ and $R_{\operatorname{logp}}$ are the $R^{2}$ values obtained respectively on the BCF and on the LogP datasets, and RMSEbcf and RMSElogp are the RMSE values obtained respectively on the BCF and on the LogP datasets. Both functions are calculated after scaling the four parameters in the range $[0,1]$ and transforming RMSEbcf and RMSElogp in their respective complements. Thus, all the four parameters had values in the range $[0,1]$ where values towards 1 mean optimality. Importantly, both DES and UTI returned values

Table 6 Best ten results for keys weights/similarity metrics combinations

\begin{tabular}{lllllllllll}
\hline $\mathbf{W}_{\mathbf{f p}}$ & $\mathbf{W}_{\mathbf{h d}}$ & $\mathbf{W}_{\mathbf{c d}}$ & $\mathbf{W}_{\mathbf{f g}}$ & Metrics & $\mathbf{B C F}^{\mathbf{2}}$ & $\mathbf{B C F}$ RMSE & LogP R & LogP RMSE & DES & $\mathbf{U T I}^{\mathbf{2}}$ \\
\hline 0.4 & 0.1 & 0.35 & 0.15 & 3 & 0.63 & 0.83 & 0.87 & 0.68 & 0.996 & 0.996 \\
0.3 & 0.15 & 0.35 & 0.2 & 3 & 0.62 & 0.84 & 0.87 & 0.67 & 0.996 & 0.996 \\
0.3 & 0.15 & 0.3 & 0.25 & 3 & 0.62 & 0.84 & 0.87 & 0.68 & 0.993 & 0.993 \\
0.3 & 0.1 & 0.35 & 0.25 & 3 & 0.62 & 0.84 & 0.87 & 0.67 & 0.992 & 0.992 \\
0.3 & 0.2 & 0.35 & 0.15 & 3 & 0.62 & 0.84 & 0.87 & 0.67 & 0.992 \\
0.3 & 0.2 & 0.3 & 0.2 & 3 & 0.62 & 0.84 & 0.87 & 0.68 & 0.992 \\
0.3 & 0.2 & 0.25 & 0.25 & 3 & 0.62 & 0.84 & 0.87 & 0.69 & 0.991 & 0.991 \\
0.4 & 0.15 & 0.3 & 0.15 & 3 & 0.62 & 0.83 & 0.86 & 0.70 & 0.989 \\
0.4 & 0.1 & 0.3 & 0.2 & 3 & 0.62 & 0.84 & 0.86 & 0.69 & 0.989 \\
0.3 & 0.05 & 0.35 & 0.3 & 3 & 0.61 & 0.85 & 0.87 & 0.67 & 0.989 \\
\hline
\end{tabular}

$\mathrm{W}_{\mathrm{xx}}$ stands for the weights of the different keys contributions (FP, HD, CD, FG, as defined in the article), Metrics for the number (id) of the non-binary similarity coefficient (as reported in Table 4), for the R2 correlation coefficient, RMSE for the root mean square error, DES for the desirability function, UTI for the utility function. 
in the range $[0,1]$, such values have been used to rank all the permutations, with higher values flagging better solutions.

Noteworthy, the rankings obtained from the desirability and the utility functions had exactly the same sorting for the top ten solutions, as reported in Table 5 .

The fingerprints found in the ten best solutions are the Extended Fingerprints, Pubchem Key and Default Fingerprint. It is interesting to note that two different approaches emerged as best solutions, as the Default and Extended fingerprints are strictly related, while Pubchem is a structural key.

For the fingerprints, it is not surprising that the Extended yield better results than the Default, as Extended are the same as default with the extension of extra bits encoding information about rings. Other fingerprints, that are similar to the Default but contain less (more generic) information such as Graph-Only or Hybridization disclose far more worse results. The best coefficients found in combination with the fingerprints are 37 (Maxwell-Pilliner), 34 (Cohen), 18 (Rogot-Goldberg), 42 (CT4), 13 (SokalSneath), 1 (simple matching).

The Pubchem key appears in the best solutions only once, combined with the similarity coefficient no. 28 (Sokal-Sneath 3).

In the second step, having selected the Extended fingerprints and the coefficient no. 37 (Maxwell-Pilliner) as the best solutions, a number of about 7200 combinations of weights and non-binary similarity coefficients has been analyzed. Similarly to the the first step, we calculated the utility and desirability values considering both the $R^{2}$ and RMSE of the read-across approach on the two datasets, and ranked the combinations. Both the rankings obtained from the desirability and the utility functions are equal with respect to the top ten best solutions, reported in Table 6.

A first result is that all the ten best solutions use the coefficient no. 3 (Bray-Curtis) for the measurement of the non-binary keys of descriptors. Subsequently, it can be easily observed that all the ten solutions have a similar distribution of the weight values. In the best solution the fingerprints block represents the most important contribution (weight of 0.4), followed by the Constitutional Descriptors block (0.35), the Functional Groups Descriptors block (0.15) and the Heteroatoms Descriptors block (0.1). This result can be interpreted as follows:

- The SI is mainly constituted by the classical fingerprint-based comparison, strongly corrected with some constitutional information like number (and type) of atoms and number (and type) of bonds; this part of the SI could be considered as the core contribution to generalizability of the SI.

- A smaller contribution of functional and heteroatoms descriptors is required to extend the information embedded in the fingerprint and constitutional descriptor blocks; we would consider this block as the part of SI which explains the "fine chemical differences" within the dataset.

\section{Conclusions}

The computation of similarities between chemical compounds is usually based on the use of common binary representations of chemical structures (i.e. 2D fingerprints) and a similarity coefficient (usually the Tanimoto distance). It has been recently demonstrated by Todeschini et al. [14] that other similarity coefficients perform better than the Tanimoto distance in terms of effectiveness for similarity-based virtual screening using simulated and real datasets. With our work, we demonstrated how achieve a higher accuracy in measures of chemical similarity by combining fingerprints with non-binary structural keys based on constitutional molecular descriptors. The basic idea is that such a combination can resolve the drawbacks of a plain fingerprint approach. Thus, we built a combined similarity index, where a fingerprint and 3 molecular descriptors based structural keys are combined with different weights. We then designed a combinatorial process to evaluate the performances of different fingerprints, different similarity coefficients, and different weighting schemes for the elements of the final index, in the context of two heterogeneous datasets.

\section{Competing interests}

The authors declare that they have no competing interests.

\section{Authors' contributions}

Conceived and designed the experiments: EB, MF and AM. Performed the experiments: MF. Wrote the paper: MF, AM and ON. All authors analyzed the data, discussed the results and commented on the manuscript. All authors have given approval to the final version of the manuscript.

\section{Acknowledgements}

We acknowledge the financial support of the LIFE + Programme, project LIFE PROSIL.

\section{Author details}

${ }^{1}$ CRS4 Biomedicine, Parco Polaris, Loc. Pixinamanna, Pula, Italy. ${ }^{2}$ Istituto di Ricerca Genetica e Biomedica (IRGB), CNR, Monserrato, Italy. ${ }^{3}$ IRCCS-Istituto di Ricerche Farmacologiche Mario Negri, Milan, Italy. ${ }^{4}$ Dipartimento di Farmacia Scienze del Farmaco, Università degli Studi di Bari "Aldo Moro", Bari, Italy.

Received: 22 May 2014 Accepted: 14 July 2014

Published online: 18 October 2014

\section{References}

1. Bender A, Glen RC: Molecular similarity: a key technique in molecular informatics. Org Biomol Chem 2004, 2:3204-3218.

2. Nikolova N, Jaworska J: Approaches to measure chemical similarity - a review. QSAR Comb Sci 2003, 22:1006-1026.

3. Bender A, Jenkins JL, Scheiber J, Sukuru SCK, Glick M, Davies JW: How similar are similarity searching methods? A principal component analysis of molecular descriptor space. J Chem Inf Model 2009, 49:108-119.

4. Willett $P$ : Similarity searching using $2 D$ structural fingerprints. Methods $\mathrm{Mol}$ Biol 2011, 672:133-158

5. Willett $P$ : Similarity-based virtual screening using 2D fingerprints. Drug Discov Today 2006, 11:1046-1053. 
6. Haranczyk M, Holliday J: Comparison of similarity coefficients for clustering and compound selection. J Chem Inf Model 2008, 48:498-508.

7. Durant JL, Leland BA, Henry DR, Nourse JG: Reoptimization of MDL keys for use in drug discovery. J Chem Inf Comput Sci 2002, 42:1273-1280.

8. Flower DR: On the properties of bit string-based measures of chemical similarity. J Chem Inf Comput Sci 1998, 38:379-386.

9. Godden X, Bajorath J: Combinatorial preferences affect molecular similarity/diversity calculations using binary fingerprints and Tanimoto coefficients. J Chem Inf Comput Sci 2000, 40:163-166.

10. Daylight Chemical Information Systems Inc. http://www.daylight.com/ dayhtml/doc/theory/theory.finger.html.

11. Tripos Inc. www.tripos.com.

12. Steinbeck C, Han Y, Kuhn S, Horlacher O, Luttmann E, Willighagen EL: The Chemistry Development Kit (CDK): an open-source java library for chemo- and bioinformatics. Chem Inf Comput Sci 2003, 43(2):493-500. doi:10.1021/ci025584y.

13. Steinbeck C, Hoppe C, Kuhn S, Floris M, Guha R, Willighagen EL, Recent Developments of the Chemistry Development Kit (CDK) : An open-source java library for chemo- and bioinformatics. Curr Pharm Des 2006, 12(17):2111-2120. (doi:10.2174/138161206777585274)].

14. Todeschini R, Consonni V, Xiang H, Holliday J, Buscema M, Willett P: Similarity coefficients for binary chemoinformatics data: overview and extendedcomparison using simulated and real data sets. $J$ Chem Inf Model 2012, 52(11):2884-2901. doi:10.1021/ci300261r. Epub 2012 Nov 7. PubMed PMID: 23078167

15. VEGA project. website: http://www vega-qsareu/.

16. Hall $L H$, Kier $L B$ : Electrotopological state indices for atom types: a novel combination of electronic, topological, and valence state information. J Chem Inf Comput Sci 1995, 35:1039-1045.

17. Klekota J, Roth FP: Chemical substructures that enrich for biological activity. Bioinformatics 2008, 24:2518-2525.

18. MACCS Structural Keys. CA (USA): Symyx Software S. R

19. National Center for Biotechnology Information: 'PubChem Substructure Fingerprint v1.3.' PubChem Data Specification Directory, 1 May 2009. $\mathrm{ftp} / /$ ftp.ncbi.n/m.nih.gov/pubchem/specifications/pubchem_fingerprints.txt (17 July 2014, date last accessed)

20. Org.openscience.cdk.fingerprint: SubstructureFingerprinter. http://pele. farmbio.uu.se/nightly/api/org/openscience/cdk/fingerprint/ SubstructureFingerprinter.html. (17 July 2014, date last accessed).

21. Talete srl, Dragon (Software for Molecular Descriptor Calculation). http://www.talete.mi.it/products/dragon_description.htm (17 July 2014 date last accessed)

22. Al Khalifa A, Haranczyk M, Holliday J: Comparison of nonbinary similarity coefficients for similarity searching, clustering and compound selection. J Chem Inf Model 2009, 49(5):1193-1201. doi:10.1021/ci8004644. PubMed PMID:19405526.

23. Pavan M, Todeschini R: Scientific Data Ranking Methods, Theory and Applications. Amsterdam: Elsevier Science; 2008. http://www.sciencedirect. com/science/book/9780444530202.

24. Nicolotti O, Carotti A: Improving quantitative structure-activity relationships through multiobjective optimization. J Chem Inf Model 2009, 49(10):2290-2302.

doi:10.1186/s13321-014-0039-1

Cite this article as: Floris et al:: A generalizable definition of chemical similarity for read-across. Journal of Cheminformatics 2014 6:39.

\section{Publish with ChemistryCentral and every scientist can read your work free of charge \\ "Open access provides opportunities to our colleagues in other parts of the globe, by allowing anyone to view the content free of charge." W. Jeffery Hurst, The Hershey Company. \\ - available free of charge to the entire scientific community \\ - peer reviewed and published immediately upon acceptance \\ - cited in PubMed and archived on PubMed Central \\ - yours - you keep the copyright \\ Submit your manuscript here: \\ http://www.chemistrycentral.com/manuscript/<smiles>c1ccccc1</smiles> Chemistry Central}

